\title{
Quasiperiodic attractors, Borel summability and the Bryuno condition for strongly dissipative systems
}

\author{
Guido Gentile ${ }^{\text {a) }}$ \\ Dipartimento di Matematica, Università di Roma Tre, Roma, I-00146, Italy \\ Michele V. Bartuccelli ${ }^{\text {b) }}$ and Jonathan H. B. Deane ${ }^{\mathrm{c})}$ \\ Department of Mathematics and Statistics, University of Surrey, Guildford, GU2 7XH, \\ United Kingdom
}

(Received 14 March 2006; accepted 23 May 2006; published online 18 July 2006)

\begin{abstract}
We consider a class of ordinary differential equations describing one-dimensional analytic systems with a quasiperiodic forcing term and in the presence of damping. In the limit of large damping, under some generic nondegeneracy condition on the force, there are quasiperiodic solutions which have the same frequency vector as the forcing term. We prove that such solutions are Borel summable at the origin when the frequency vector is either any one-dimensional number or a twodimensional vector such that the ratio of its components is an irrational number of constant type. In the first case the proof given simplifies that provided in a previous work of ours. We also show that in any dimension $d$, for the existence of a quasiperiodic solution with the same frequency vector as the forcing term, the standard Diophantine condition can be weakened into the Bryuno condition. In all cases, under a suitable positivity condition, the quasiperiodic solution is proved to describe a local attractor. (C) 2006 American Institute of Physics.
\end{abstract}

[DOI: $10.1063 / 1.2213790]$

\section{INTRODUCTION}

In this paper we pursue the study started in Refs. 6 and 2. We consider one-dimensional systems with a quasiperiodic forcing term in the presence of strong damping, described by ordinary differential equations of the form

$$
\varepsilon \ddot{x}+\dot{x}+\varepsilon g(x)=\varepsilon f(\boldsymbol{\omega} t),
$$

where $\boldsymbol{\omega} \in \mathbb{R}^{d}$ is the frequency vector, $g(x)$ and $f(\boldsymbol{\psi})$ are functions analytic in their arguments, with $f$ quasiperiodic, i.e.,

$$
f(\boldsymbol{\psi})=\sum_{\boldsymbol{\nu} \in Z^{d}} e^{i \boldsymbol{\nu} \cdot \boldsymbol{\psi}} f_{\boldsymbol{\nu}}
$$

with average $\langle f\rangle=f_{0}$, and $\varepsilon>0$ is a real parameter, physically representing the inverse of the damping coefficient. With $\cdot$ we are denoting the scalar product in $\mathbb{R}^{d}$. A Diophantine condition is assumed on $\boldsymbol{\omega}$ for $d>1$, that is

$$
|\boldsymbol{\omega} \cdot \boldsymbol{\nu}| \geqslant C_{0}|\boldsymbol{\nu}|^{-\tau} \quad \forall \boldsymbol{\nu} \in \mathbb{Z}^{d} \backslash\{\boldsymbol{0}\},
$$

where $|\boldsymbol{\nu}|=|\boldsymbol{\nu}|_{1} \equiv\left|\nu_{1}\right|+\cdots+\left|\nu_{d}\right|$, and $C_{0}$ and $\tau$ are positive constants. The set of vectors satisfying the condition (1.3) is nonvoid for $\tau \geqslant d-1$ and is of full measure for $\tau>d-1$. For $d=1$ we denote

\footnotetext{
${ }^{\text {a)} E l e c t r o n i c ~ m a i l: ~ g e n t i l e @ m a t . u n i r o m a 3 . i t ~}$

${ }^{b}$ Electronic mail: m.bartuccelli@ surrey.ac.uk

${ }^{c)}$ Electronic mail: j.deane@surrey.ac.uk
} 
the vectors without boldface; in that case $\omega$ will be called the frequency number.

In Ref. 6 we show that, under the nondegeneracy condition

$$
\exists c_{0} \in \mathbb{R} \quad \text { such that } g\left(c_{0}\right)=f_{0} \text { and } g^{\prime}\left(c_{0}\right) \neq 0,
$$

the system (1.1) admits a quasiperiodic solution $x(t ; \varepsilon)$ with the same frequency vector as the forcing. Such a solution can be obtained by a suitable summation of the formal power series

$$
x_{0}(t ; \varepsilon):=\sum_{k=0}^{\infty} \varepsilon^{k} x^{(k)}(t), \quad x^{(k)}(t)=\sum_{\boldsymbol{\nu} \in \mathbb{Z}^{d}} e^{i \boldsymbol{\nu} \cdot \boldsymbol{\omega} t} x_{\boldsymbol{\nu}}^{(k)},
$$

which solves the equations of motion order by order. For $d=1$ (periodic forcing) the series (1.4) is Borel summable in $\varepsilon$. In Ref. 2 we also show that if $g^{\prime}\left(c_{0}\right)>0$, for any $d$ such a solution is locally an attractor. In some cases, for instance if $g(x)=x^{2 p+1}, p \in \mathbb{N}$, and $f_{0}>0$, the attractor is global.

In this paper we first give a different (simpler) proof of Borel summability in the periodic case (Sec. II), then we prove that the formal series for the solution turns out to be Borel summable also for $d=2$ and $\tau=1$ (Sec. III); this corresponds to frequency vectors with components such that their ratios are irrational numbers of constant type (i.e., numbers with bounded partial quotients in their continued fraction expansion). The proof does not rely on Nevanlinna-type theorems, ${ }^{7,8}$ but consists in checking directly that the conditions for the formal series of the solution to be Borel summable are satisfied, and follows the same strategy introduced in Ref. 4 to investigate Borel summability of lower-dimensional tori.

Finally in Sec. IV we show how to relax the Diophantine condition. We show that, in order to have the same results on existence and attractivity of the quasiperiodic solution, one can take $\boldsymbol{\omega}$ to be a Bryuno vector, that is one can assume that, by defining

$$
B(\boldsymbol{\omega})=\sum_{n=0}^{\infty} \frac{1}{2^{n}} \log \frac{1}{\alpha_{n}(\boldsymbol{\omega})}, \quad \alpha_{n}(\boldsymbol{\omega})=\inf _{|\boldsymbol{\nu}| \leqslant 2^{n}}|\boldsymbol{\omega} \cdot \boldsymbol{\nu}|,
$$

then $\boldsymbol{\omega}$ satisfies the Bryuno condition $B(\boldsymbol{\omega})<\infty$. More formal statements will be given in the next sections.

\section{BOREL SUMMABILITY FOR $d=1$}

First of all let us recall the definition of Borel summability. ${ }^{8}$ Let $f(\varepsilon)=\sum_{n=1}^{\infty} a_{n} \varepsilon^{n}$ be a formal power series (which means that the sequence $\left\{a_{n}\right\}_{n=1}^{\infty}$ is well defined). We say that $f(\varepsilon)$ is Borel summable if

(1) $B(p):=\sum_{n=1}^{\infty} a_{n} p^{n} / n$ ! converges in some circle $|p|<\delta$,

(2) $B(p)$ has an analytic continuation to a neighborhood of the positive real axis, and

(3) $g(\varepsilon)=\int_{0}^{\infty} e^{-p / \varepsilon} B(p) \mathrm{d} p$ converges for some $\varepsilon>0$.

Then the function $B(p)$ is called the Borel transform of $f(\varepsilon)$, and $g(\varepsilon)$ is the Borel sum of $f(\varepsilon)$. Moreover if the integral defining $g(\varepsilon)$ converges for some $\varepsilon_{0}>0$ then it converges in the circle $\operatorname{Re} \varepsilon^{-1}>\operatorname{Re} \varepsilon_{0}^{-1}$. A function which admits the formal power series expansion $f(\varepsilon)$ is called Borel summable if $f(\varepsilon)$ is Borel summable; in that case the function equals the Borel sum $g(\varepsilon)$.

Theorem 2.1: Consider the system (1.1) for $d=1$, and assume that the nondegeneracy condition (1.4) is fulfilled. There exists $\varepsilon_{0}>0$ such that for $|\varepsilon|<\varepsilon_{0}$ there is a periodic solution $x(t ; \varepsilon)$ which has the same frequency number as the forcing term and is Borel summable in $\varepsilon$ at the origin. If $g^{\prime}\left(c_{0}\right)>0$ such a solution describes a local attractor.

Proof: We consider explicitly the case $g(x)=x^{2}$ in $(1.1)$, which corresponds to the varactor equation extensively studied in Refs. 6, 1, and 2; the general case can be easily dealt with by reasoning as in Sec. VII of Ref. 6. In Ref. 6 we proved that the formal power series (1.5) is well defined and that to any order $k$ one has 


$$
\left|x_{\nu}^{(k)}\right| \leqslant A_{1} \varepsilon_{2}^{-k} k !, \quad\left|x^{(k)}(t)\right| \leqslant A_{1} \varepsilon_{2}^{-k} k !,
$$

for suitable constants $A_{1}$ and $\varepsilon_{2}$ [cf. formula (4.5) in Ref. 6]. This means that the first condition, in the definition of Borel summability, is satisfied, with $\delta=\varepsilon_{2}$.

In Ref. 6 we also proved that the formal power series can be summed, and gives a function

$$
x(t ; \varepsilon)=\sum_{k=0}^{\infty} \sum_{\nu \in Z} e^{i \omega \nu t} x_{\nu}^{[k]},
$$

which is real-analytic and periodic in $t$, and analytic in $\varepsilon$ in a suitable domain tangent to the imaginary axis at the origin. The coefficients $x_{v}^{[k]}$ can be written as

$$
x_{\nu}^{[k]}=\sum_{\theta \in \mathcal{T}_{k, \nu}} \operatorname{Val}(\theta), \quad \operatorname{Val}(\theta)=\left(\prod_{\ell \in L(\theta)} g_{\ell}\right)\left(\prod_{v \in E(\theta) \cup V(\theta)} F_{v}\right),
$$

where the symbols are defined as in Sec. V of Ref. 6. We briefly recall the basic definitions and notations, with the purpose of making self-consistent the discussion; reference should be made to Ref. 6 for further details.

A tree $\theta$ is a graph, that is a connected set of points and lines, with no cycle, such that all the lines are oriented toward a unique point (root) which has only one incident line (root line). All the points in a tree except the root are denoted nodes. The orientation of the lines in a tree induces a partial ordering relation $(\leqslant)$ between the nodes. Given two nodes $v$ and $w$, we shall write $w \leqslant v$ every time $v$ is along the path (of lines) which connects $w$ to the root. We call $E(\theta)$ the set of endpoints in $\theta$, that is the nodes which have no entering line. The endpoints can be represented either as white bullets or as black bullets; we denote with $E_{W}(\theta)$ and $E_{B}(\theta)$ the set of white bullets and the set of black bullets, respectively. With each endpoint $v$ we associate a mode label $\nu_{v}$ $\in \mathbb{Z}$, such that $\nu_{v}=0$ if $v \in E_{W}(\theta)$ and $\nu_{v} \neq 0$ if $v \in E_{B}(\theta)$. We denote with $L(\theta)$ the set of lines in $\theta$. Since $\ell$ is uniquely identified with the point $v$ which it leaves, we may write $\ell=\ell_{v}$. With each line $\ell$ we associate a momentum label $\nu_{\ell} \in \mathbb{Z}$. The modes of the endpoints and and the momenta of the lines are related as follows: if $\ell=\ell_{v}$ one has

$$
\nu_{\ell}=\sum_{i=1}^{s_{v}} \nu_{\ell_{i}}=\sum_{w \in E_{B}(\theta): w \leqslant v} \nu_{w}
$$

where $s_{v}$ denotes the number of lines entering $v$ [one has $s_{v}=2$ if $g(x)=x^{2}$ in (1.1), otherwise $s_{v}$ $\geqslant 2]$, and $\ell_{1}, \ldots, \ell_{s_{v}}$ are the lines entering $v$. We denote by $V(\theta)$ the set of vertices in $\theta$, that is the set of points which have at least one entering line. We set $V_{0}(\theta)=\left\{v \in V(\theta): \nu_{\ell_{v}}=0\right\}$. We call equivalent two trees which can be transformed into each other by continuously deforming the lines in such a way that they do not cross each other. Let $\mathcal{T}_{k, \nu}$ be the set of inequivalent trees of order $k$ and total momentum $\nu$, that is the set of inequivalent trees $\theta$ such that $|V(\theta)|+\left|E_{B}(\theta)\right|=k$ and the momentum of the root line is $\nu$. We associate with each line $\ell$ a propagator

$$
g_{\ell}= \begin{cases}1 /\left(\left(i \omega \nu_{\ell}\right)\left(1+i \varepsilon \omega \nu_{\ell}\right)\right), & \nu_{\ell} \neq 0, \\ 1, & \nu_{\ell}=0,\end{cases}
$$

with each vertex $v$ a node factor

$$
F_{v}= \begin{cases}-\varepsilon, & v \notin V_{0}(\theta), \\ -1 / 2 c_{0}, & v \in V_{0}(\theta),\end{cases}
$$

and with each endpoint $v$ a node factor 


$$
F_{v}= \begin{cases}c_{0}, & v \in E_{W}(\theta), \\ \varepsilon f_{\nu_{v}}, & v \in E_{B}(\theta) .\end{cases}
$$

Then (2.3) says that each coefficient $x_{\nu}^{[k]}$ is given by the sum over all trees of order $k$ and total momentum $\nu$ of the corresponding values.

It is more convenient to slightly change the definition of node factors and propagators, by associating the factor $\varepsilon$ with the propagator $g_{\ell}$ of the line $\ell$ coming out from $v$ and not with $v$ itself. In this way the propagator of any line with $\ell$ momentum $\nu_{\ell} \neq 0$ is

$$
g_{\ell}=g\left(\omega \nu_{\ell} ; \varepsilon\right), \quad g(x ; \varepsilon)=\frac{\varepsilon}{i x(1+i \varepsilon x)},
$$

and the only dependence on $\varepsilon$ in $\operatorname{Val}(\theta)$ is through the product of propagators with nonvanishing momentum. [Note that $g(x ; \varepsilon)$ in $(2.8)$ has a completely different meaning with respect to the function $g(x)$ appearing in (1.1). The same caveat applies to the propagators $g^{[n]}(x ; \varepsilon)$ in Sec. III.]

The function (2.8) is Borel summable, and its Borel transform is easily computed to be

$$
g_{B}(x ; p)=\frac{e^{-i p x}}{i x} \Rightarrow\left|g_{B}(x ; p)\right| \leqslant \frac{e^{|\operatorname{Im} p||x|}}{|x|} .
$$

Moreover $g_{B}(x ; p)$ is an entire function in $p$, and the integral $\int_{0}^{\infty} e^{-p / \varepsilon} g_{B}(x ; p) \mathrm{d} p$ converges (absolutely) for all $\varepsilon>0$.

For any tree $\theta \in \mathcal{T}_{k, \nu}$ the Borel transform of $\operatorname{Val}(\theta)$ is given by a constant times the Borel transform of the product of the propagators with nonzero momentum. One has

$$
(\operatorname{Val}(\theta))_{B}(p)=\left(\prod_{\ell \in L_{0}(\theta)} g_{\ell}\right)\left(\prod_{v \in E(\theta) \cup V(\theta)} F_{v}\right)\left(\left(\prod_{\ell \in L_{2}(\theta)} g_{\ell}\right)_{B}(p)\right)
$$

where we have called $L_{0}(\theta)$ is the set of lines in $L(\theta)$ with zero momentum, and we have set $L_{2}(\theta)=L(\theta) \backslash L_{0}(\theta)$ (cf. Sec. IV of Ref. 6). The Borel transform appearing in (2.10) equals the convolution of the Borel transforms of the propagators with nonzero momentum, so that it can be bounded as

$$
\left|\left(\prod_{\ell \in L_{2}(\theta)} g_{\ell}\right)_{B}(p)\right| \leqslant \prod_{\ell \in L_{2}(\theta)}{ }^{*}\left|g_{B}\left(\omega \nu_{\ell} ; p\right)\right| \leqslant\left(\prod_{\ell \in L_{2}(\theta)} \frac{1}{\left|\omega \nu_{\ell}\right|}\right) \frac{|p|^{k-1}}{(k-1) !} \exp \left(|\operatorname{Im} p| \max _{\ell \in L_{2}(\theta)}\left|\omega \nu_{\ell}\right|\right),
$$

where $\Pi^{*}$ denotes the convolution product, and $|\omega|<\left|\omega \nu_{\ell}\right|<|\omega| \Sigma_{v \in E_{B}(\theta)}\left|\nu_{v}\right|$; cf. Remarks (4) to (6) after Definition 1 in Ref. 3 for properties of the Borel transforms we are using here.

Therefore, for $p$ in any strip $\Sigma_{\sigma}=\{p \in \mathrm{C}:|\operatorname{Im} p|<\sigma\}$ of the real axis, we have

$$
\left|\prod_{v \in E_{B}(\theta)} F_{v}\right| \exp \left(|\operatorname{Im} p| \max _{\ell \in L_{2}(\theta)}\left|\omega \nu_{\ell}\right|\right) \leqslant F^{\left|E_{B}(\theta)\right|} \prod_{v \in E_{B}(\theta)} e^{-\xi\left|\nu_{v}\right| / 2},
$$

provided $|\omega| \sigma<\xi / 2$, and summability over the Fourier labels in (2.3) is assured. The sum over $k$ in (2.2) produces a quantity bounded proportionally to the exponential $e^{\Gamma|p|}$, for some positive constant $\Gamma$. A comparison with Ref. 6 shows that $\Gamma=1 / \varepsilon_{0}$, where $\varepsilon_{0}$ is the same as in the statement of the theorem. In particular the Borel transform $x_{B}(t ; p)$ of the series $(2.2)$ turns out to have an analytic continuation to the strip $\Sigma_{\sigma}$, and admits there the bound $\left|x_{B}(t ; p)\right| \leqslant C e^{\Gamma|p|}$, for a suitable constant $C$. Hence the integral 


$$
g(t ; \varepsilon):=\int_{0}^{\infty} e^{-p / \varepsilon} x_{B}(t ; p) \mathrm{d} p
$$

absolutely converges provided $0<\varepsilon<\varepsilon_{0}$. So also the last two conditions for the formal series of $x(t ; \varepsilon)$ to be Borel summable are satisfied.

That the solution $x(t ; \varepsilon)$ describes a local attractor, under the further condition $g^{\prime}\left(c_{0}\right)>0$, follows from the analysis performed in Ref. 2 .

Note that, because of the analyticity properties of $x_{B}(t ; p)$, it follows, as a consequence of Nevanlinna's theorem, ${ }^{8}$ that the function defined by the integral (2.13) is analytic in the circle $C_{R}=\left\{\varepsilon \in \mathrm{C}: \operatorname{Re} \varepsilon^{-1}>R^{-1}\right\}$, with $R=\varepsilon_{0}$, and satisfies the bound

$$
g(t ; \varepsilon)=\sum_{k=0}^{N-1} \varepsilon^{k} x^{(k)}(t)+\Re_{N}(\varepsilon), \quad\left|\Re_{N}(\varepsilon)\right| \leqslant A B^{N} N !|\varepsilon|^{N},
$$

with constants $A$ and $B$ independent of $N$. This is consistent with Proposition 5.3 of Ref. 6 .

\section{BOREL SUMMABILITY FOR $d=2$ AND $\tau=1$}

In the case of quasiperiodic forcing terms for $d=2$ we obtain the following result.

Theorem 3.1: Consider the system (1.1) for $d=2$, and assume that $\boldsymbol{\omega}$ satisfies the Diophantine condition (1.3) with $\tau=1$ and that the nondegeneracy condition (1.4) is fulfilled. There exists $\varepsilon_{0}$ $>0$ such that for $|\varepsilon|<\varepsilon_{0}$ there is a quasiperiodic solution $x(t ; \varepsilon)$ which has the same frequency vector as the forcing term and is Borel summable at the origin. If $g^{\prime}\left(c_{0}\right)>0$ such a solution describes a local attractor.

Proof: Again we discuss explicitly the case $g(x)=x^{2}$ in (1.1). Let $\psi$ be a nondecreasing $C^{\infty}$ function defined in $\mathbb{R}_{+}$, such that

$$
\psi(u)= \begin{cases}1 & \text { for } u \geqslant 1, \\ 0 & \text { for } u \leqslant 1 / 2,\end{cases}
$$

and set $\chi(u):=1-\psi(u)$. Define, for all $n \in \mathbb{Z}_{+}, \chi_{n}(u):=\chi\left(2^{n} C_{0}^{-1} u / 4\right)$ and $\psi_{n}(u):=\psi\left(2^{n} C_{0}^{-1} u / 4\right)$.

With each line $\ell$ with zero momentum we associate a scale label $n_{\ell}=-1$, while with each line with nonzero momentum we associate (arbitrarily) a scale label $n_{\ell} \in \mathbb{Z}_{+}=\{0\} \cup \mathbb{N}$. Then we can define cluster and self-energy clusters as in Refs. 4 and 6. A cluster $T$ on scale $n$ is a maximal set of points and lines connecting them such that all the lines have scales $n^{\prime} \leqslant n$ and there is at least one line with scale $n$. The lines entering the cluster $T$ and the possible line coming out from it (unique if existing at all) are called the external lines of the cluster $T$. Given a cluster $T$ on scale $n$, we shall denote by $n_{T}=n$ the scale of the cluster; we call $V(T), E(T), E_{W}(T), E_{B}(T)$, and $L(T)$ the set of vertices, of endpoints, of white endpoints, of black endpoints, and of lines of $T$, respectively. We call self-energy cluster any cluster $T$ such that $T$ has only one entering line $\ell_{T}^{2}$ and one exiting line $\ell_{T}^{1}$, and one has $\Sigma_{v \in E_{B}(T)} \boldsymbol{\nu}_{v}=\mathbf{0}$. With each line $\ell$ with momentum $\boldsymbol{\nu}_{\ell}$ and scale $n_{\ell}$ we associate a renormalized propagator $g_{\ell}=g^{\left[n_{\ell}\right]}\left(\boldsymbol{\omega} \cdot \boldsymbol{\nu}_{\ell} ; \varepsilon\right)$, still to be defined. On the contrary the node factors are defined as in the previous case (with the only trivial difference that now $\boldsymbol{\nu}_{v}$, replacing $\nu_{v}$, is a $d$-dimensional vector).

Define the self-energy value $\mathcal{V}_{T}(\boldsymbol{\omega} \cdot \boldsymbol{\nu} ; \varepsilon)$ in terms of the renormalized propagators and node factors as

$$
\mathcal{V}_{T}(\boldsymbol{\omega} \cdot \boldsymbol{\nu} ; \varepsilon)=\left(\prod_{\ell \in L(T)} g^{\left[n_{\ell}\right]}\left(\boldsymbol{\omega} \cdot \boldsymbol{\nu}_{\ell} ; \varepsilon\right)\right)\left(\prod_{v \in E(T) \cup V(T)} F_{v}\right),
$$

where $\boldsymbol{\nu}$ is the momentum of both the external lines of $T$.

We proceed as in Sec. VI of Ref. 6, with the only two differences that we perform a preliminary summation by including the contribution $-2 \varepsilon c_{0}$ (arising from the self-energy graphs on scale 
-1) into the propagator $g^{[0]}(x ; \varepsilon)$, and —as in the periodic case of Sec. II-we associate the factors $\varepsilon$ to the propagators with nonzero momentum. Therefore we define [see the comment after (2.8) in Sec. II]

$$
g^{[0]}(x ; \varepsilon)=\frac{\varepsilon \psi_{0}(|x|)}{i x(1+i \varepsilon x)-2 \varepsilon c_{0}}, \quad M^{[0]}(x ; \varepsilon)=\varepsilon \sum_{k=1}^{\infty} \sum_{T \in S_{k, 0}^{\mathcal{R}}} \mathcal{V}_{T}(x ; \varepsilon)
$$

whereas the propagators on scale $n \geqslant 1$ are defined as in Ref. 6 , again with a factor $\varepsilon$ appearing in the numerator of the propagators with nonzero momentum; this means that one has

$$
\begin{gathered}
g^{[n]}(x ; \varepsilon)=\frac{\varepsilon \chi_{0}(|x|) \cdots \chi_{n-1}(|x|) \psi_{n}(|x|)}{i x(1+i \varepsilon x)-\mathcal{M}^{[n-1]}(x ; \varepsilon)}, \\
\mathcal{M}^{[n]}(x ; \varepsilon)=\sum_{p=1}^{n} \chi_{0}(|x|) \cdots \chi_{p-1}(|x|) \chi_{n}(|x|) M^{[p]}(x ; \varepsilon), \quad M^{[n]}(x ; \varepsilon)=\varepsilon \sum_{k=1}^{\infty} \sum_{T \in \mathcal{S}_{k, n}^{\mathcal{R}}} \mathcal{V}_{T}(x ; \varepsilon),
\end{gathered}
$$

where the set of renormalized self-energy clusters $\mathcal{S}_{k, n}^{\mathcal{R}}$ is defined and the set of self-energy clusters $T$ on scale $n_{T}=n$ and of order $k$ [that is with $|V(T)|+\left|E_{B}(T)\right|=k$ ]. With respect to Refs. 6 and 4 a further factor $\varepsilon$ appears in $M^{[n]}(x ; \varepsilon), n \geqslant 0$, simply because there is one such factor per node (vertex or endpoint) with exiting line carrying a nonzero momentum-cf. Sec. 6 in Ref. 4-and we are associating the factors $\varepsilon$ with the lines instead of the nodes.

An easy computation gives, for the Borel transform of $g^{[0]}(x ; \varepsilon)$,

$$
g_{B}^{[0]}(x ; p)=\frac{\psi_{0}(|x|)}{i x} \exp \left(-i p\left(x-2 \frac{c_{0}}{x}\right)\right) \Rightarrow\left|g_{B}^{[0]}(x ; p)\right| \leqslant \frac{1}{|x|} e^{\left(|x|+2\left|c_{0}\right| / x \mid\right)|\operatorname{Im} p|} .
$$

If we set, for $n \geqslant 0$,

$$
\widetilde{g}^{[n]}(x ; \varepsilon)=\frac{\varepsilon}{i x(1+i \varepsilon x)-\mathcal{M}^{[n-1]}(x ; \varepsilon)} \quad \forall|x| \leqslant 2^{-(n-1)} C_{0},
$$

and define $M^{[n]}(x ; \varepsilon)=\mathcal{M}^{[n]}(x ; \varepsilon)-\mathcal{M}^{[n-1]}(x ; \varepsilon)$, we obtain the recursive equations

$$
\left(\widetilde{g}^{[n]}(x ; \varepsilon)\right)^{-1}=\left(\widetilde{g}^{[n-1]}(x ; \varepsilon)\right)^{-1}-\chi_{0}(|x|) \cdots \chi_{n-1}(|x|) \varepsilon^{-1} M^{[n-1]}(x ; \varepsilon), \quad n \geqslant 1 .
$$

By using these equations we can prove inductively the bound

$$
\left|\widetilde{g}_{B}^{[n]}(x ; p)\right| \leqslant \frac{K_{0}}{|x|} e^{\left(c_{n}+c_{n}^{\prime}|x|^{-1 / 2}\right)|p|+\kappa_{0}|\operatorname{Im} p|\left(d_{n}|x|+d_{n}^{\prime}|x|^{-1}\right)}
$$

where $K_{0}$ and $\kappa_{0}$ are two constants, and the sequences $\left\{c_{n}\right\}_{n=0}^{\infty},\left\{c_{n}^{\prime}\right\}_{n=0}^{\infty},\left\{d_{n}\right\}_{n=0}^{\infty},\left\{d_{n}^{\prime}\right\}_{n=0}^{\infty}$ are to be found.

The proof proceeds as in Appendix A1 of Ref. 3. Set $x_{\ell}=\boldsymbol{\omega} \cdot \boldsymbol{\nu}_{\ell}$, and call $L_{0}(T)$ and $L_{2}(T)$ the set of lines in $L(T)$ with zero momentum and the set $L_{2}(T)=L(T) \backslash L_{0}(T)$, respectively. First we use the inductive bound to obtain 


$$
\begin{aligned}
\left|\left(\frac{M^{[N]}(x ; \varepsilon)}{\varepsilon}\right)_{B}\right| \leqslant & \sum_{k=2}^{\infty} \sum_{T \in \mathcal{S}_{k, N-1}^{\mathcal{R}}}\left(\prod_{\ell \in L_{0}(T)}\left|g_{\ell}\right|\right)\left(\prod_{v \in E(T) \cup V(T)}\left|F_{v}\right|\right) \\
& \times\left(\prod_{\ell \in L_{2}(T)} * \frac{K_{0}}{\left.\left.\left|x_{\ell} e^{\left(c_{n_{\ell}}+c_{n}^{\prime}\right.}\right| x_{\ell}\right|^{-1 / 2}\right)|p|+\kappa_{0}\left(d_{n_{\ell}}\left|x_{\ell}\right|+d_{n_{\ell}}^{\prime}\left|x_{\ell}\right|^{-1}\right)|\operatorname{Im} p|}\right) \\
\leqslant & \left(\prod_{v \in E_{B}(\theta)} e^{-\xi\left|\nu_{v}\right| \mid} \sum_{k=2}^{\infty} \Gamma^{k} \frac{|p|^{k-2}}{(k-2) !} e^{\left(c_{N-1}+c_{N-1}^{\prime} 2^{N / 2}\right)|p|+\kappa_{0} d_{N-1}^{\prime} 2^{N}|\operatorname{Im} p|},\right.
\end{aligned}
$$

where $D_{0}=\Gamma^{2}, r_{N}=\Gamma+c_{N-1}+\Gamma_{0} c_{N-1}^{\prime} 2^{N / 2}$, for some $N$-independent constant $\Gamma_{0}$. The bound in the last line of (3.9) has been obtained by using part of the exponential decay (say one-fourth) of the node factors associated with the endpoints to control the exponent $\kappa_{0} d_{N-1} \max _{\ell \in L_{2}(T)}\left|x_{\ell}\right|$, provided $d_{N-1}<d$ for some $N$-independent constant $d$ and $|\operatorname{Im} p| \leqslant \sigma$, with $\sigma$ small enough, more precisely $\kappa_{0} \sigma d|\omega|<\xi / 4$.

By explicitly performing the sum over $k$ we obtain from (3.6),

$$
\left|\left(\frac{M^{[N]}(x ; \varepsilon)}{\varepsilon}\right)_{B}\right| \leqslant D_{0} e^{r_{N}|p|} e^{-\xi_{0} 2^{N}}
$$

where we have used the bound $\Sigma_{\nu \in E_{B}(T)}\left|\boldsymbol{\nu}_{v}\right| \geqslant \Gamma_{1} 2^{N}$, for a suitable constant $\Gamma_{1}$-see formula (7.12) of Ref. 4-and again part of the exponential decay (say another one-fourth) of the node factors associated with the endpoints to control the exponent $\kappa_{0} d_{N-1}^{\prime} 2^{N}|\operatorname{Im} p|$, provided again $d_{N-1}^{\prime}<d^{\prime}$ for some $N$-independent constant $d^{\prime}$ and $\kappa_{0} d^{\prime} \sigma<\xi \Gamma_{1} / 4$; in particular one finds $\xi_{0}=\Gamma_{1} \xi / 4$.

Then, by using (3.10) and, once more, the inductive bound, we obtain from (3.7),

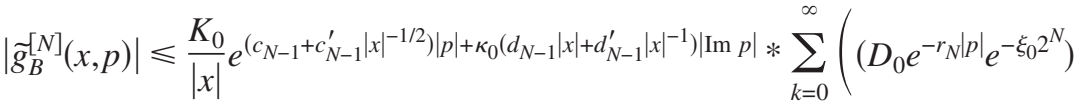

$$
\begin{aligned}
& \left.*\left(\frac{K_{0}}{|x|} e^{\left(c_{N-1}+c_{N-1}^{\prime}|x|^{-1 / 2}\right)|p|+\kappa_{0}\left(d_{N-1}|x|+d_{N-1}^{\prime}|x|^{-1}\right)|\operatorname{Im} p|}\right)\right)^{* k},
\end{aligned}
$$

with $a^{* k}=a * a * \cdots * a(k$ times $)$. This gives

$$
\left|\widetilde{g}_{B}^{[N]}(x, p)\right| \leqslant \frac{K_{0}}{|x|} \sum_{k=0}^{\infty} \frac{1}{(2 k) !}\left(\frac{K_{0}|p|^{2}}{|x|} D_{0} e^{-\xi_{0} 2^{N}}\right)^{K} e^{\left(r_{N}+c_{N-1}^{\prime}|x|^{-1 / 2}\right)|p|+\kappa_{0}\left(d_{N}|x|+d_{N}^{\prime}|x|^{-1}\right)|\operatorname{Im} p|},
$$

which implies the bound (3.5) for $n=N$, with $c_{N}=r_{N}=\Gamma+c_{N-1}+\Gamma_{0} c_{N-1}^{\prime} 2^{N / 2}, \quad c_{N}^{\prime}=c_{N-1}^{\prime}$ $+\sqrt{K_{0} D_{0} e^{-\xi_{0} 0^{N}}}, d_{N}=d_{N-1}$ and $d_{N}^{\prime}=d_{N-1}^{\prime}$. In particular one has $d_{N}=d=1$ and $d_{N^{\prime}}=d^{\prime}=2\left|c_{0}\right|$, so that there exists a constant $c>0$ such that $\max \left\{c_{n} 2^{-n / 2}, c_{n}^{\prime}, d_{n}, d_{n^{\prime}}\right\} \leqslant c$ for all $n \geqslant 0$.

The bounds (3.8) for the Borel transforms of the propagators can be used to obtain a bound on the Borel transform $x_{B}(t ; p)$ of $x(t ; \varepsilon)$. We omit the details, which can be derived exactly as in Appendix A1 of Ref. 3. Eventually one finds the bound

$$
\left|x_{B}(t ; p)\right| \leqslant C_{1} e^{C_{2}|p|^{2}},
$$

for suitable constants $C_{1}$ and $C_{2}$. Again, the bound (3.13) and the analyticity properties of $x_{B}(t ; p)$ implies that $x(t ; \varepsilon)$ is Borel summable, and it can be written for $\varepsilon>0$ as

$$
x(t ; \varepsilon)=\int_{0}^{\infty} e^{-p / \varepsilon} x_{B}(t ; p) \mathrm{d} p,
$$

in terms of its Borel transform.

As in the case $d=1$ the last statement of the theorem has been proved in Ref. 2 . 
In the general case $g(x) \neq x^{2}$ in (1.1) the quantity $2 c_{0}$ must be replaced with $g^{\prime}\left(c_{0}\right)$, with $g^{\prime}\left(c_{0}\right) \neq 0$ by hypothesis. Then the discussion proceeds as in Sec. VII of Ref. 6.

Note also that in the case $d=2$ and $\tau=1$ the Borel transform is still defined in a strip around the real axis, but it does not satisfy any more an exponential bound like in the case $d=1$ (at least the argument given above does not provide an estimate of this kind). Thus, we cannot apply Nevanlinna's theorem to prove Borel summability. ${ }^{7,8}$

\section{BRYUNO FREQUENCY VECTORS}

Let $\boldsymbol{\omega} \in \mathbb{R}^{d}$ be a Bryuno vector. This means that $B(\boldsymbol{\omega})<\infty$, with $B(\boldsymbol{\omega})$ defined in (1.6).

Theorem 4.1: Consider the system (1.1) for any $d \geqslant 2$, and assume that $\boldsymbol{\omega}$ satisfies the Bryuno condition $B(\omega)<\infty$ and that the nondegeneracy condition (1.4) is fulfilled. There exists $\varepsilon_{0}>0$ such that for all real $|\varepsilon|<\varepsilon_{0}$ there is a quasiperiodic solution with frequency vector $\boldsymbol{\omega}$. If $g^{\prime}\left(c_{0}\right)>0$ such a solution describes a local attractor.

For simplicity's sake we discuss the case $g(x)=x^{2}$ and $\varepsilon \in \mathbb{R}$, but the analysis can be easily generalized to any analytic function $g$ [provided the nondegeneracy condition (1.4) is satisfied]. Furthermore the solution can be showed to extend to a function analytic in $\varepsilon$ in the domain $\mathcal{C}_{R}$ defined in Sec. VI of Ref. 6 (cf. Fig. 16 in Ref. 6).

Let $\psi(x)$ be the nondecreasing $C^{\infty}$ function defined in (3.1) and set $\chi(x):=1-\psi(x)$. Define, for all $n \in \mathbb{Z}_{+}, \chi_{n}(x):=\chi\left(\alpha_{n}^{-1}(\boldsymbol{\omega}) x / 4\right)$ and $\psi_{n}(x):=\psi\left(\alpha_{n}^{-1}(\boldsymbol{\omega}) x / 4\right)$.

Set $g^{[-1]}(x ; \varepsilon)=1$ and $M^{[-1]}(x ; \varepsilon)=0$, and define iteratively $g^{[n]}(x ; \varepsilon)$ and $M^{[n]}(x ; \varepsilon)$ as done in the case of Diophantine vectors. This means that for $n=0$ we can define $g^{[0]}(x ; \varepsilon)$ and $M^{[0]}(x ; \varepsilon)$ as in (3.3), while for $n \geqslant 1$ we define

$$
\begin{gathered}
g^{[n]}(x ; \varepsilon)=\frac{\varepsilon \chi_{0}(|x|) \cdots \chi_{n-1}(|x|) \psi_{n}(|x|)}{i x(1+i \varepsilon x)-\mathcal{M}^{[n-1]}(x ; \varepsilon)}, \\
\mathcal{M}^{[n]}(x ; \varepsilon)=\sum_{p=0}^{n} \chi_{0}(|x|) \cdots \chi_{p}(|x|) M^{[p]}(x ; \varepsilon), \quad M^{[n]}(x ; \varepsilon)=\varepsilon \sum_{k=1}^{\infty} \sum_{T \in \mathcal{S}_{k, n}^{\mathcal{R}}} \mathcal{V}_{T}(x ; \varepsilon),
\end{gathered}
$$

where $\mathcal{S}_{k, n}^{\mathcal{R}}$ is the set of renormalized self-energy clusters $T$ on scale $n$ and of order $k$, and the self-energy value $\mathcal{V}_{T}(x ; \varepsilon)$ is defined as in (3.2). Note that we are using the same definitions of Sec. III, in particular we are associating the factors $\varepsilon$ with the propagators rather than with the nodes (contrary to what done in Ref. 6). So far the only difference with respect to the case of the standard Diophantine condition concerns the multiscale decomposition: the factors $2^{n} C_{0}^{-1}$ appearing in $\chi_{n}$ and $\psi_{n}$ are substituted with $\alpha_{n}^{-1}(\omega)$.

Lemma 4.2: Assume that the renormalized propagators up to scale $n-1$ can be bounded as

$$
\left|g^{\left[n_{\ell}\right]}\left(\boldsymbol{\omega} \cdot \boldsymbol{\nu}_{\ell} ; \varepsilon\right)\right| \leqslant C^{-1} \alpha_{n_{\ell}}^{-\beta}(\boldsymbol{\omega})
$$

for some positive constants $\beta$ and $C$. Then for all $p \leqslant n-1$ the number $N_{p}(\theta)$ of lines on scale $p$ in any renormalized tree $\theta$ and the number $N_{p}(T)$ of lines on scale $p$ in any renormalised self-energy cluster $T$ are bounded both by

$$
N_{p}(\theta) \leqslant K 2^{-p} \sum_{v \in E_{B}(\theta)}\left|\boldsymbol{\nu}_{v}\right|, \quad N_{p}(T) \leqslant K 2^{-p} \sum_{v \in E_{B}(T)}\left|\boldsymbol{\nu}_{v}\right|,
$$

for some positive constant $K$. If $|\varepsilon|<\varepsilon_{0}$, with $\varepsilon_{0}$ small enough, then for all $p \leqslant n-1$ one has

$$
\left|M^{[p]}(x ; \varepsilon)\right| \leqslant D_{1}|\varepsilon|^{2} e^{-D_{2} 2^{p}}, \quad\left|\partial_{x} M^{[p]}(x ; \varepsilon)\right| \leqslant D_{1}|\varepsilon|^{2} e^{-D_{2} 2^{p}},
$$

for some positive constants $D_{1}$ and $D_{2}$. Only the constant $D_{1}$ depends on $\beta$. The constant $\varepsilon_{0}$ can be written as $\varepsilon_{0}=C_{1} \alpha_{n_{0}}^{\beta}$, with $n_{0}(\boldsymbol{\omega}, \beta)$ such that 


$$
K \beta \sum_{n=n_{0}+1}^{\infty} \frac{1}{2^{n}} \log \frac{1}{\alpha_{n}(\boldsymbol{\omega})} \leqslant \frac{\xi}{4},
$$

and $C_{1}$ a positive constant dependending on $C$ but not on $\beta$.

Proof: The lemma can be proved by reasoning as in Refs. 4 and 5. We simply sketch the proof, and omit the details. First of all note that, if we define $n(\boldsymbol{\nu})=\left\{n \in \mathbb{Z}_{+}: 2^{n-1}<|\boldsymbol{\nu}| \leqslant 2^{n}\right\}$ then one has $|\boldsymbol{\omega} \cdot \boldsymbol{\nu}| \geqslant \alpha_{n(\boldsymbol{\nu})}(\boldsymbol{\omega})$. Moreover $n^{\prime}>n$ implies $\alpha_{n^{\prime}}(\boldsymbol{\omega}) \leqslant \alpha_{n}(\boldsymbol{\omega})$, and $\alpha_{n^{\prime}}(\boldsymbol{\omega})<\alpha_{n}(\boldsymbol{\omega})$ implies $n^{\prime}>n$. Set $M(\theta)=\Sigma_{v \in E_{B}(\theta)}\left|\boldsymbol{\nu}_{v}\right|$ and $M(T)=\Sigma_{v \in E_{B}(T)}\left|\boldsymbol{\nu}_{v}\right|$. The bound on $N_{p}(\theta)$ is obtained by proving by induction on the order of the renormalized tree that if $N_{p}(\theta) \neq 0$ then $N_{p}(\theta) \leqslant 22^{-p} M(\theta)$ -1 Then, given a renormalized self-energy cluster $T \in \mathcal{S}_{k, n}^{\mathcal{R}}$, one proves first that $M(T)>2^{n-1}$, hence, again by induction, that if $N_{p}(T) \neq 0$ then $N_{p}(T) \leqslant 22^{-p} M(T)-1$. Therefore (4.3) is proved. An important property is that if a cluster $T$ has two external lines, with momenta $\boldsymbol{\nu}$ and $\boldsymbol{\nu}^{\prime}$, respectively, with $\boldsymbol{\nu} \neq \boldsymbol{\nu}^{\prime}$, both on scales greater or equal to $n$, so that $|\boldsymbol{\omega} \cdot \boldsymbol{\nu}| \leqslant \alpha_{n-1}(\boldsymbol{\omega}) / 4$ and $|\boldsymbol{\omega} \cdot \boldsymbol{\nu}| \leqslant \alpha_{n-1}(\boldsymbol{\omega}) / 4$, then one has $\left|\boldsymbol{\omega} \cdot\left(\boldsymbol{\nu}-\boldsymbol{\nu}^{\prime}\right)\right|<\alpha_{n-1}(\boldsymbol{\omega})$, hence $n\left(\boldsymbol{\nu}-\boldsymbol{\nu}^{\prime}\right) \geqslant n$, so that $M(T) \geqslant \mid \boldsymbol{\nu}$ $-\boldsymbol{\nu}^{\prime} \mid>2^{n-1}$. For details we refer to Ref. 5 .

The bounds (4.4) are obtained by exploiting the just mentioned bound on $M(T)$ and half the exponential decay factors $e^{-\xi\left|\boldsymbol{\nu}_{v}\right|}$ associated with the vertices and endpoints internal to $T$ to derive the factors $e^{-D_{2} 2^{p}}$, with $D_{2}$ independent of $\beta$, and by using the fact that any self-energy cluster $T$ contributing to $M^{[p]}(x ; \varepsilon)$ must be of order at least 2 to derive the factors $|\varepsilon|^{2}$.

Then for any $n_{0} \in \mathbb{N}$ and for any tree $\theta$, we can bound each propagator on scale up to $n_{0}$ with $C^{-1} \alpha_{n_{0}}^{-\beta}(\boldsymbol{\omega})$ and the product of propagators on scale greater than $n_{0}$ with

$$
\prod_{n=n_{0}+1}\left(C^{-1} \alpha_{n}^{-\beta}(\boldsymbol{\omega})\right)^{N_{n}(\theta)}=C^{-\Sigma_{n=n_{0}+1}^{\infty} N_{n}(\theta)} \exp \left(\beta M(\theta) \sum_{n=n_{0}+1}^{\infty} \frac{1}{2^{n}} \log \frac{1}{\alpha_{n}(\boldsymbol{\omega})}\right),
$$

so that, by choosing $n_{0}$ according to (4.5), the last exponential in (4.6) is controlled by half the exponential decay factor $e^{-\xi M(T)}$ arising from the node factors. Then the sum of the values of all trees of order $k$ is bounded by $\left(C^{-1} C^{\prime} \alpha_{n_{0}}^{-\beta}\right)^{k}$, for a suitable constant $C^{\prime}$ - taking into account all the constants other than $C$ and the sums over the trees. Hence also the assertion about the dependence of $\varepsilon_{0}$ on $\alpha_{n_{0}}(\boldsymbol{\omega})$ follows, and the proof of the lemma is complete.

As in Ref. 6, to prove existence of the quasiperiodic solution we need the following result, which together with Lemma 4.2 provides the proof of Theorem 4.1.

Lemma 4.3: For real $\varepsilon$ small enough the renormalized propagators satisfy the bounds (4.2) with $\beta=1$. For $\varepsilon$ in the domain $\mathcal{C}_{R}$ in Fig. 16 of Ref. 6 they satisfy the bounds (4.2) with $\beta=2$.

Proof: The proof can be carried out exactly as in Ref. 6. Indeed it is enough to show that the propagators $g^{[n]}(x ; \varepsilon)$ can be bounded proportionally to $|x|^{-\beta}$, for $\varepsilon$ small enough in a suitable domain, and this follows from Lemmata 6.2 to 6.5 of Ref. 6, independently on the particular Diophantine condition assumed on $\boldsymbol{\omega}$.

The proof of the theorem is completed if we show that the quasiperiodic solution is a local attractor if $g^{\prime}\left(c_{0}\right)>0$. But this can be proved as in the case of Diophantine frequency vectors, by reasoning as in Ref. 2: indeed the only property that we need for the argument given in Ref. 2 to work is the existence of the quasiperiodic solution.

\footnotetext{
${ }^{1}$ Bartuccelli, M. V., Deane, J. H. B., Gentile, G., and Marsh, L., "Invariant sets for the varactor equation,” Proc. R. Soc. London, Ser. A 462, 439-457 (2006).

${ }^{2}$ Bartuccelli, M. V., Deane, J. H. B., and Gentile, G., Globally and locally attractive solutions for quasiperiodically forced systems, J. Math. Anal. Appl. (in press).

${ }^{3}$ Costin, O., Gallavotti, G., Gentile, G., and Giuliani, A., Borel summability and Lindstedt series, Commun. Math. Phys. (in press).

${ }^{4}$ Gentile, G., "Quasi-periodic solutions for two-level systems," Commun. Math. Phys. 242, 221-250 (2003).

${ }^{5}$ Gentile, G., Resummation of perturbation series and reducibility for Bryuno skew-product flows, J. Stat. Phys. (in press).
} 
${ }^{6}$ Gentile, G., Bartuccelli, M. V., and Deane, J. H. B., "Summation of divergent series and Borel summability for strongly dissipative differential equations with periodic or quasiperiodic forcing terms," J. Math. Phys. 46, 062704 (2005).

${ }^{7}$ Nevanlinna, F., "Zur Theorie der Asymptotischen Potenzrehen," Ann. Acad. Sci. Fenn., Ser. A 12, 1-18 (1916).

${ }^{8}$ Sokal, A. D., "An improvement of Watson's theorem on Borel summability," J. Math. Phys. 21, 261-263 (1980). 